\title{
SALPINGECTOMY AT THE TIME OF HYSTERECTOMY FOR BENIGN GYNAECOLOGIC DISEASE: A COMPARISON OF SURGICAL APPROACHES
}

\author{
Obermair, $\mathrm{HM}^{1}$; Borg, $\mathrm{E}^{2}$ \\ 1 - Liverpool Hospital, South-West Sydney Local Health District, Sydney, NSW, Australia \\ 2 - Caboolture Hospital, Metro North Health District, Brisbane, QLD, Australia
}

\section{Introduction}

- Emerging evidence suggests that a significant proportion of high-grade serous ovarian cancers originate in the fallopian tubes (1)

- Retrospective cohort studies demonstrate a potential $50 \%$ reduction in risk of ovarian cancer in those who have undergone bilateral salpingectomy (2)

- Outside of a woman's reproductive period, there is no known benefit for retaining the fallopian tubes

- As such, the RANZCOG Guidelines recommend that 'consideration be given to bilateral salpingectomy at the time of hysterectomy for benign gynaecological disease' (3)

\section{Aims \& Objectives}

- This study aimed to identify the practices of Obstetricians \& Gynaecologists in Australia and New Zealand with regards to salpingectomy at the time of hysterectomy for benign gynaecologic disease

\section{Methods}

- This study was performed through a retrospective review of data collected for clinical audit

- An exemption from formal ethics review was provided by the Royal Brisbane and Women's Hospital

- Data were collected from SurgicalPerformance.com, an Australian service providing web-based surgical outcomes reviews

- Patients were included if the primary surgical procedure was a hysterectomy for benign indication. Patients were excluded if their hysterectomy was performed outside of Australia or New Zealand.

- Cases occurring prior to the inclusion of 'Bilateral Salpingectomy' as a selectable option on data entry were excluded. Only operations occurring after the 1st July 2009 were included, as this was the date of the RANZCOG guideline recommending consideration of bilateral salpingectomy

- We compared the incidence of bilateral salpingectomy and bilateral salpingo-oophorectomy between groups of different surgical approaches using Chi Square analysis

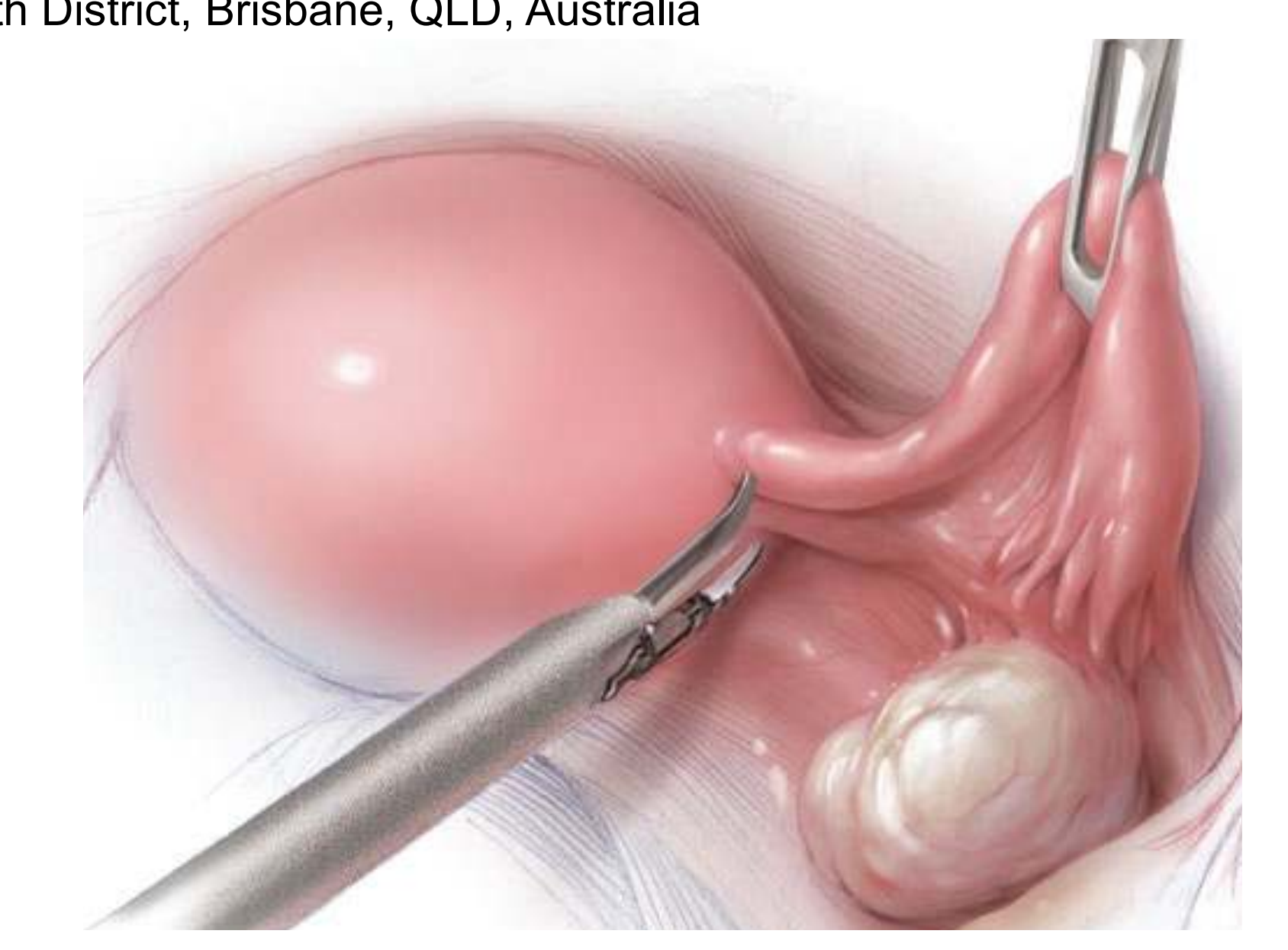

Results

- Average age $=47+/-10.1$ (standard deviation)

- Of 11, 477 records of patients who had a hysterectomy, 6,608 cases met all inclusion criteria and were included in analysis

Patient characteristics

- BMI and ASA scores were well balanced between the surgical groups

- Patients who had a vaginal hysterectomy were slightly older and more likely to have had a hysterectomy as part of a pelvic floor repair

\section{Outcomes}

- Bilateral salpingectomy or bilateral salpingo-oophorectomy were performed in $58 \%$ of patients undergoing hysterectomy

- There was a significantly lower rate of salpingectomy at vaginal hysterectomy $(13 \%)$ compared to open abdominal $(65 \%)$, laparoscopic $(70 \%)$, laparoscopic assisted vaginal $(78 \%)$ or robotic $(73 \%)$ hysterectomies

- Rates of salpingectomy with vaginal hysterectomy were significantly lower (Chi-square 1487.9, p<0.001), compared to other approaches

\section{Conclusion}

- The results of our study demonstrate that patients were less likely to have a salpingectomy if they had a vaginal hysterectomy compared to other open or minimally invasive surgical approaches to hysterectomy

- Of adnexal surgeries performed at the time of hysterectomy, bilateral salpingectomy was more common in women under the age of 51 years, and

bilateral salpingo-oophrectomy was more frequently performed in women above age 51 years of age.

- Previous research surveying gynaecologists about their practices demonstrates a similar, although less pronounced, difference (4)

Limitations of this study

- Self-reported data

- Cannot confirm whether this cohort is representative of O\&Gs in Australia/New Zealand

- Reports only a small subset of 30,000 hysterectomies performed in Australia each year

- Unknown how many patients had a salpingectomy prior to hysterectomy

While the data presented here are solely hypothesis generating, they justify further prospective research into barriers to salpingectomy when a vaginal approach to hysterectomy is taken.

\section{References}

(1) Kindelberger, D.W., Lee, Y., Miron, A., et al. Intraepithelial carcinoma of the fimbria and pelvic serous carcinoma: Evidence for a causal relationship. Am J Surg Pathol 2007; 31: 161-169.

(2) Yoon, S.H., Kim, S.N., Shim, S.H., et al. Bilateral salpingectomy can reduce the risk of ovarian cancer in the general population: A meta-analysis. Eur J Cancer 2016; 55: 38-46.

(3) The Royal Australian and New Zealand College of Obstetricians and Gynaecologists. Melbourne: Managing the adnexae at the time of hysterectomy for benign gynaecological disease, 2009 [Cited March 9 2019]. Available from https://www.ranzcog.edu.au/RANZCOG SITE/media/RANZCOGMEDIA/Women\%27s\%20Health/Statement\%20and\%20guidelines/Clinical\%20-\%20Gynaecology/Managing-the-adnexae-at-the-time-of-hysterectomy-(C-Gyn-25)March18.pdf?ext=.pdf

(4) Kapurubandara, S., Qin, V., Gurram, D., et al. Opportunistic bilateral salpingectomy during gynaecological surgery for benign disease: A survey of current Australian practice. Aust NZ J Obstet Gynaecol 2015; 55: 606-611. 